\title{
OBSTRUÇÃO INTESTINAL NO IDOSO
}

\section{Intestinal obstruction in the elderly}

\author{
Maria Aparecida Coelho de Arruda HENRY, Mauro Masson LERCO, \\ Walmar Kerche de OLIVEIRA, Leandro Teodoro CRIPPA, Paulo Augusto Zeratti MONTEIRO, \\ Ismael Augusto Silva LOMBARDI, Gabriel Doreto RODRIGUES
}

ABCDDV/564

Henry MACA, Lerco MM, Oliveira WK, Crippa LT, Monteiro PAZ, Lombardi IAS, Rodrigues GD. Obstrução intestinal no idoso. ABCD Arq Bras Cir Dig 2007;20(4):225-9

RESUMO - Racional - O envelhecimento da população é realidade sentida na vivência diária, levando à necessidade associada de prover cuidados médicos eletivos e de emergência a um número cada vez maior de idosos. Objetivo - Avaliar os aspectos clínicos, terapêuticos e evolutivos de pacientes idosos com obstrução intestinal atendidos no Hospital das Clínicas da Faculdade de Medicina de Botucatu - UNESP. Métodos - Análise retrospectiva de pacientes internados no período de janeiro de 2002 a dezembro de 2006 . Foram estudados 50 pacientes, 24 homens e 26 mulheres, com idade média de 74,7 7,4 anos, analisando-se a diferenciação entre obstrução funcional e orgânica; a freqüência das causas obstrutivas; a mortalidade; a incidência dos sinais e sintomas; e correlação laboratorial. Resultados - O quadro obstrutivo intestinal teve as seguintes causas: brida (38\%), câncer de colon (24\%), hérnias (12\%), impactacão fecal (12\%), carcinomatose (10\%) e volvo da sigmóide (4\%). A mortalidade foi de $18 \%$ associada à complicações infecciosas em todos os pacientes. Conclusões - a) A distinção entre a obstrução intestinal funcional e orgânica oferece dificuldade no idoso; b) o câncer do colon constitui-se em importante causa de obstrução intestinal com quadro obstrutivo já na primeira manifestação clínica desse tumor; c) bridas e hérnias são igualmente importantes na etiologia da obstrução intestinal; d) os sinais clínicos obstrutivos são poucos evidentes no idoso e a leucometria é mais fidedigna para este diagnóstico.

DESCRITORES - Obstrução intestinal, idoso, tratamento.

\section{INTRODUÇÃO}

A população brasileira vem apresentando nas últimas cinco décadas mudanças nos níveis de mortalidade e fecundidade, as quais vêm caindo a uma velocidade nunca vista anteriormente ${ }^{19}$. Essa mudança de comportamento populacional - à qual dá-se o nome de transição demográfica ${ }^{19,27}$ - é a responsável pelo envelhecimento da população.

A medida que o número de pessoas que atingem idade avançada continua a crescer, existe necessidade associada de prover cuidados médicos eletivos e de emergência a um número cada vez maior de pacientes idosos ${ }^{13}$.

Dentre as causas de internação hospitalar, a obstrução intestinal ocupa posição de destaque, sendo responsável por $3 \%$ das admissões ${ }^{2,22}$.

Com relação ao atendimento do paciente idoso com obstrução intestinal, existem questões importantes que devem ser elucidadas, tais como distinção entre obstrução funcional ou mecânica, simples ou estrangulada e deter-

Trabalho realizado no Departamento de Cirurgia e Ortopedia da Faculdade de Medicina de Botucatu - UNESP, Botucatu, São Paulo, Brasil.

Endereço para correspondência: Maria Aparecida Coelho de Arruda Henry, e-mail: rhenry@ibb.unesp.br minação do tempo ideal de operação ${ }^{30}$.

O objetivo deste trabalho é analisar os aspectos clínicos, terapêuticos e evolutivos de idosos admitidos com diagnóstico de obstrução intestinal.

\section{MÉTODOS}

Foram analisados os registros médicos de 50 pacientes idosos com diagnóstico de obstrução intestinal, internados na Enfermaria de Gastrocirurgia no período de janeiro de 2002 a dezembro de 2006. Como critério de inclusão foi o de pacientes com idade igual ou superior a 60 anos.

O diagnóstico de obstrução intestinal foi baseado nos dados de história clínica, exame físico, exame radiológico do abdome nas posições ortostática e decúbito (Figura 1), dosagens laboratoriais (eletrólitos, hemograma) e quando necessário tomografia computadorizada do abdome. Para avaliar se os pacientes com estrangulamento intestinal exibiam os sinais considerados clássicos desta afecção, foram avaliados neste grupo 3 parâmetros, a saber temperatura corporal (TC), freqüência cardíaca (FC) e leucometria (L) 


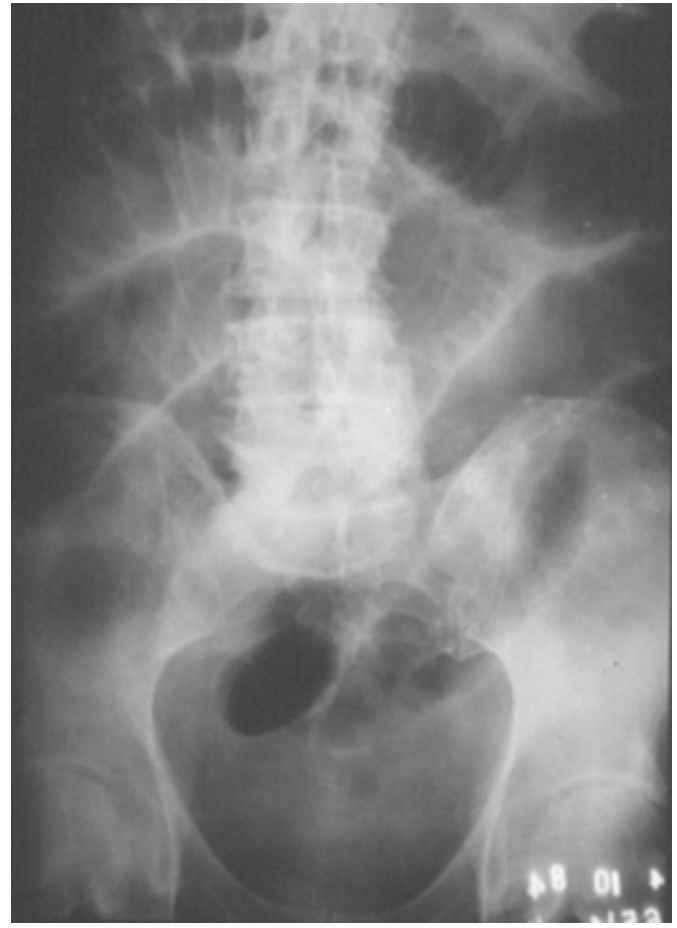

FIGURA 1 - Exame radiológico do abdome com o paciente em decúbito: alças de intestino delgado dilatadas

\section{RESULTADOS}

Foram estudados 50 pacientes, sendo 24 homens e 26 mulheres, com idades variando entre 62 e 92 anos (média de 74,7 $\pm 7,4$ anos). Doze apresentavam idade superior a 80 anos, e 2 mais de noventa (Tabela 1).

TABELA 1 - Dados demográficos de pacientes idosos com obstrução intestinal, com e sem estrangulamento intestinal

\begin{tabular}{lccc}
\hline Sexo & N & Com estrangulamento & Sem estrangulamento \\
\hline Homens & 24 & 4 & 20 \\
Mulheres & 26 & 2 & 24 \\
\hline Total & 50 & 6 & 44 \\
\hline & & & \\
\hline Idade & $\mathbf{N}$ & Com estrangulamento & Sem estrangulamento \\
\hline $60-69$ & 12 & 0 & 12 \\
$70-79$ & 26 & 5 & 21 \\
$80-89$ & 10 & 1 & 9 \\
$90-99$ & 2 & 0 & 2 \\
\hline Total & 50 & 6 & 44 \\
\hline
\end{tabular}

A duração das queixas variou entre 2 e 15 dias, com média de 4,6 $\pm 3,9$ dias. Dentre os sintomas referidos pelos pacientes, a dor abdominal acompanhada de distensão, vômitos e parada de eliminação de gases e fezes foram os mais comuns.

Em conformidade com a rotina do serviço, os pacientes foram inicialmente submetidos a tratamento conservador, visando a descompressão do trato gastrointestinal, exceção feita para aqueles com sinais de irritação peritoneal. Esta conduta consiste em colocação de sonda nasogástrica, hidratação parenteral, sonda vesical e, quando necessário, sonda retal.

A adoção desta conduta foi benéfica para 21 pacientes, com restabelecimento do hábito intestinal. Dentre eles, 16 referiam operações abdominais prévias, sendo a colecistectomia, apendicectomia e retosigmoidectomia as mais citadas. Neste grupo ficou estabelecido como diagnóstico etiológico da obstrução a brida pós-operatória. Todavia cinco pacientes com evolução favorável frente ao tratamento conservador negavam tal antecedente, sendo aventada a hipótese de impactação fecal.

Vinte e sete pacientes foram submetidos a tratamento cirúrgico, por falta de resposta frente às medidas conservadoras. $\mathrm{O}$ achado cirúrgico mais observado foi o câncer do colon (12 pacientes) (Figura 2), seguido de hérnia (6 pacientes), sendo duas inguinais, duas femorais, uma interna e uma incisional. Cinco pacientes apresentavam carcinomatose, 3 com bridas e 1 com impactação fecal (Tabela 2).

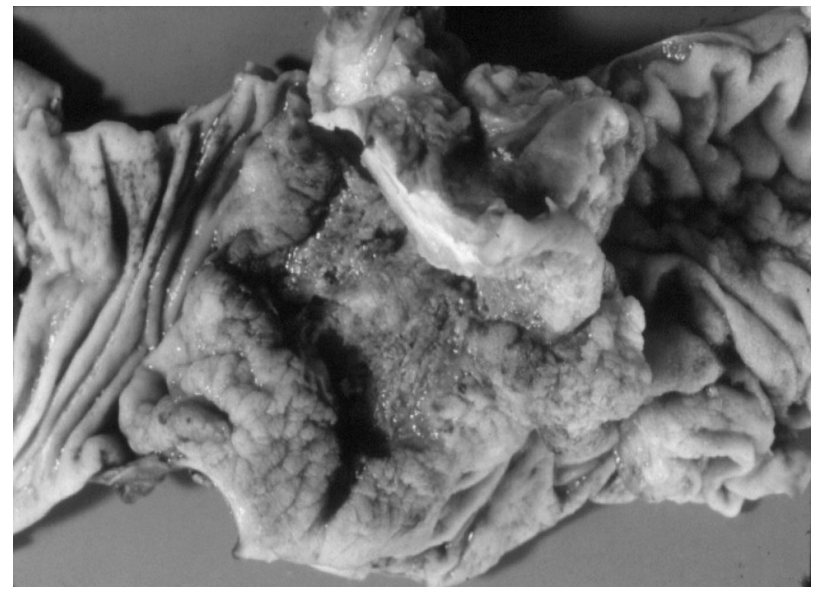

FIGURA 2 - Lesão estenosante do ceco, cujo exame anatomopatológico demonstrou adenocarcinoma

TABELA 2 - Causas da obstrução intestinal

\begin{tabular}{lccc}
\hline Causas & N & Com estrangulamento & Sem estrangulamento \\
\hline Brida & 19 & 1 & 18 \\
Câncer do cólon & 12 & 0 & 12 \\
Hérnia & 6 & 5 & 1 \\
Impactação fecal & 6 & 0 & 6 \\
Carcinomatose & 5 & 0 & 5 \\
Volvo do sigmóide & 2 & 0 & 2 \\
\hline Total & 50 & 6 & 44 \\
\hline
\end{tabular}

Em dois pacientes foi realizado o diagnóstico de volvo de sigmóide, sendo que a distorção endoscópica foi benéfica para um deles. O outro faleceu antes do procedimento.

Nesta casuística 9 pacientes apresentaram evolução desfavorável, sendo os óbitos decorrentes de complicações infecciosas (18\%). As causas da obstrução intestinal neste 
grupo foram a doença neoplásica em 4, hérnia estrangulada em 1, brida em 3 e volvo do sigmóide em 1 paciente (Tabela 3). A idade média dos pacientes que faleceram foi mais elevada que daqueles que apresentaram boa evolução $(78,8$ $\pm 8,4 \times 73,8 \pm 6,9)$, com tendência a significância estatística $(\mathrm{P}=0,065)$. A duração das queixas entre os pacientes com má evolução foi de $5,6 \pm 6,1$ dias, enquanto que nos demais foi de 4,3 $\pm 3,3(\mathrm{P}=0,40)$ (Tabela 4).

TABELA 3 - Causas de mortalidade na obstrução intestinal do idoso

\begin{tabular}{ccccc}
\hline N & Idade & Sexo & Causa do óbito & Causa da obstrução \\
\hline 1 & 68 & M & Pneumonia & Carcinomatose \\
2 & 89 & M & Septicemia & Carcinomatose \\
3 & 83 & M & Pneumonia & N.colon transverso avançado \\
4 & 78 & M & Septicemia & N.colon avançado \\
5 & 89 & M & Septicemia & Brida \\
6 & 81 & M & Pneumonia & Brida \\
7 & 77 & M & Septicemia & Hérnia incisional estrangulada \\
8 & 79 & F & Septicemia & Volvo de sigmóide \\
9 & 64 & M & Pneumonia & Brida \\
\hline
\end{tabular}

TABELA 4 - Média e desvio-padrão referentes à idade (anos) e tempo de duração das queixas (dias) de pacientes idosos com obstrução intestinal

\begin{tabular}{lccc}
\hline Variável & G1 & G2 & Valor de $\boldsymbol{P}$ \\
\hline Idade & $78,8 \pm 8,4$ & $73,8 \pm 6,9$ & 0,065 \\
Tempo & $5,6 \pm 6,1$ & $4,3 \pm 3,3$ & 0,40 \\
\hline
\end{tabular}

$\mathrm{G} 1=$ pacientes que faleceram

G2= pacientes com evolução favorável

Estrangulamento intestinal foi observado em 6 pacientes (12\%), sendo que 2 deles faleceram (33,3\%). Esta complicação foi diagnosticada em 5 com hérnia e 1 com brida. Em relação a temperatura corporal, ela variou entre $35,4^{\circ} \mathrm{C}$ e $37^{\circ} \mathrm{C}$ (média de $36,2^{\circ} \mathrm{C}$ ). A freqüência cardíaca média neste grupo foi de 83 batimentos por minuto (variação de 70 a 120 batimentos por minuto). A contagem de leucócitos

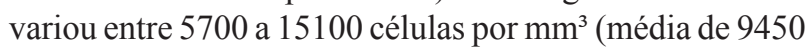
células por $\mathrm{mm}^{3}$ ) (Tabela 5 ).

TABELA 5 - Valores da temperatura corporal $\left(\mathrm{TC}-{ }^{\circ} \mathrm{C}\right)$, freqüência cardíaca (FC - batimentos/minutos) e leucometria $\left(\mathrm{L}-\right.$ células $\left./ \mathrm{mm}^{3}\right)$ em pacientes idosos com estrangulamento intestinal

\begin{tabular}{cccccccc}
\hline N & Idade & Sexo & Causa & TC & FC & L & Evolução \\
\hline 1 & 70 & M & Hérnia femoral & 35,4 & 70 & 15.100 & Boa \\
2 & 79 & M & Hérnia inguinal & 37 & 120 & 14.400 & Boa \\
3 & 73 & M & Hérnia interna & 36 & 80 & 6.500 & Boa \\
4 & 79 & F & Hérnia inguinal & 36,5 & 78 & 5.700 & Boa \\
5 & 88 & F & Brida & 36,2 & 80 & 10.200 & Óbito \\
6 & 77 & M & Hérnia incisional & 36,2 & 74 & 4.800 & Óbito \\
\hline
\end{tabular}

\section{DISCUSSÃO}

Foram avaliados neste levantamento 50 pacientes idosos, considerados como tal aqueles indivíduos com 60 anos ou mais, consideração esta inferior à referida por vários autores ${ }^{1,13,14,29,33}$. Esta diferença é decorrente do conceito de idoso estabelecido pela Organização Mundial de Saúde $(1984)^{27}$, que leva em consideração o grau de desenvolvimento do pais de origem do paciente. Assim, nos paises em desenvolvimento o sexagenário já é considerado idoso, fato que não ocorre na Europa e Estados Unidos.

Com relação a distribuição dos pacientes segundo o gênero, foi observado nesta série equivalência ( 24 homens e 26 mulheres), semelhante ao referido por Greene et al. ${ }^{14}$.

Em estudo realizado em 107 doentes com idades variando entre 70 e 93 anos, Zadeh et al. ${ }^{33}$ observaram como principal causa de obstrução intestinal a brida pósoperatória (50\%). Este resultado foi encontrado também na presente pesquisa, onde $38 \%$ dos pacientes apresentaram tal diagnóstico. Todavia outros autores, que também estudaram pacientes idosos, assinalaram como causa mais comum de obstrução intestinal a hérnia inguinal, femoral ou umbilical ${ }^{14,29}$. Em casuística constituída de adultos, porém não exclusivamente de idosos, a brida pós-operatória tem sido apontada como a causa mais importante de obstrução intestinal $^{3,6,9,16,21,22,24,28}$.

Dos 19 pacientes com brida analisados nesta série, 16 apresentaram remisão completa dos sintomas após o tratamento conservador, todavia 3 necessitaram de tratamento cirúrgico, sendo que todos faleceram. A lise das bridas foi realizada por abordagem laparotômica, embora a via laparoscópica seja recomendada por vários autores ${ }^{6,25}$.

A segunda causa de obstrução intestinal nesta casuística foi o câncer do colon, sendo o ceco o local mais acometido, seguido do sigmóide. Nesta eventualidade várias condutas têm sido preconizadas, desde ressecção com anastomose primária até procedimentos conservadores como colostomia ou colocação de stents metálicos auto-expansivos ${ }^{7,17,20}$. A escolha da conduta depende do local da lesão e das condições gerais do paciente. Para os tumores localizados no cólon direito, a conduta preconizada há vários anos tem sido a hemicolectomia com anastomose primária, procedimento realizado em 5 dos pacientes desta série, com bons resultados. Todavia, ela tem sido adotada também para as lesões do cólon esquerdo, com bons resultados ${ }^{17,20}$. Koruth et al. ${ }^{18}$ recomendam a realização de irrigação colônica intraoperatória, com redução significativa da morbimortalidade. Em cinco pacientes deste grupo a lesão estava localizada no cólon esquerdo. Em quatro foram realizadas ressecção e anastomose primária e em 1, colostomia. A evolução de todos foi satisfatória. Em 2 pacientes a neoplasia apresentava-se em estado avançado e ambos faleceram.

Os distúrbios da atividade motora gastrointestinal dos idosos têm sido relatados por vários autores ${ }^{5,8,15,31}$. A constipação intestinal crônica é sintoma comum, referido por $24 \%$ a $40 \%$ dos indivíduos desta faixa etária ${ }^{11,32}$, decorrente de erros alimentares, principalmente ingestão deficiente de fibras, associados à atividade física inadequada e uso 
de medicamentos que interferem na motilidade intestinal. A maior complicação da constipação intestinal dos idosos é a impactação fecal ${ }^{10}$, observada em 6 pacientes desta pesquisa (12\%). O tratamento desta complicação consiste na remoção do bolo fecal através de enemas, conduta adotada nos pacientes desta série, com resultado favorável em 5 deles. Em apenas 1 paciente houve necessidade de remoção cirúrgica do sigmóide, associada a procedimento de Hartman.

A hérnia tem sido relatada como importante causa de obstrução intestinal ${ }^{14,29}$, todavia neste levantamento ela foi diagnosticada em apenas 6 pacientes (12\%). Fato que causou surpresa foi o encontro de 5 pacientes com estrangulamento intestinal neste grupo de 6 pacientes com hérnia. O diagnóstico desta complicação é suspeitado frente a parâmetros clínicos que indiquem a resposta inflamatória sistêmica: febre, taquicardia e leucocitose ${ }^{33}$. A análise da Tabela 5 evidencia que dos 3 parâmetros estudados, apenas a contagem de leucócitos mostrou alterações em 2 pacientes. Um deles apresentou leucocitose ( 14.400 células por $\mathrm{mm}^{3}$ ) e outro leucopenia (4.800 células por $\mathrm{mm}$ ), o último com evolução fatal. Miyauchi et al. ${ }^{23}$ referem que em 30 pacientes com obstrução intestinal, nos com estrangulamento houve correlação positiva entre os parâmetros clínicos acima assinalados e a extensão da necrose intestinal, mas a idade média dos pacientes era de 68,4 anos, enquanto que no presente relato, a idade média dos com hérnia e estrangulamento intestinal era de 75,6 anos.

Segundo Gleckman e Hilbert ${ }^{12}$ a alteração do estado mental pode ser a única manifestação clínica de pacientes idosos com bacteremia.

Tais considerações enfatizam a dificuldade de se diagnosticar o estrangulamento intestinal em idosos, conferindo ao cirurgião a responsabilidade de indicar o tratamento da hérnia precocemente, se possível, em caráter eletivo ${ }^{3,16}$.

Cinco pacientes desta série encontravam-se em período pós-operatório tardio de operação oncológica abdominal e foram submetidos inicialmente a tratamento conservador, visando a descompressão intestinal. Tal conduta, todavia, não atingiu o objetivo, motivo pelo qual indicou-se laparotomia exploradora que evidenciou recidiva tumoral avançada em todos, inviabilizando a ressecção da lesão. Dois pacientes deste grupo faleceram.

O volvo do sigmóide foi observado em apenas 2 pacientes desta série (4\%), índice inferior ao referido por Moreira Jr et al. ${ }^{24}$. Na casuística estudada por estes autores os pacientes são oriundos de região endêmica para a doença de Chagas, onde o megacolon chagásico acomete grande parte da população. Além disso, o serviço médico dirigido pelos autores constitui centro de referência nacional para o tratamento das manifestações digestivas dessa doença. A distorção endoscópica do volvo foi realizada com sucesso em um dos pacientes desta série.

Dos 50 pacientes analisados neste levantamento, 9 evoluíram para sepse, insuficiência de múltiplos órgãos e óbito. Esta complicação pode ser explicada por translocação bacteriana, pois os portadores de obstrução intestinal apresentam alguns fatores, tais como proliferação bacteriana na alça intestinal ocluída, aumento da permeabilidade da barreira mucosa e deficiência de imunidade, que favorecem o seu desenvolvimento ${ }^{26}$. Além disso, as comorbidades próprias da senectude podem ter contribuído para o desfecho fatal, haja visto que neste grupo a idade média era superior a observada nos pacientes que apresentaram evolução favorável.

\section{CONCLUSÕES}

a) A distinção entre a obstrução intestinal funcional e orgânica oferece dificuldade no idoso; b) o câncer do colon constitui-se em importante causa de obstrução intestinal com quadro obstrutivo já na primeira manifestação clínica; c) bridas e hérnias são igualmente importantes na etiologia da obstrução intestinal; d) os sinais clínicos obstrutivos são poucos evidentes no idoso e a leucometria é mais fidedigna para este diagnóstico.

Henry MACA, Lerco MM, Oliveira WK, Crippa LT, Monteiro PAZ, Lombardi IAS, Rodrigues GD. Intestinal obstruction in the elderly. ABCD Arq Bras Cir Dig 2007;20(4):225-9.

ABSTRACT - Background - Population aging is a reality that is experienced daily, leading to an associated need for providing elective and emergency medical care to an increasing number of elderly individuals. Aim - To evaluate clinical, therapeutic and developmental aspects of patients with intestinal obstruction assisted at the University Hospital of the Botucatu School of Medicine - UNESP. Methods - Retrospective analysis of patients hospitalized from January 2002 to December 2006. A group of fifty patients comprising 24 males and 26 females at a mean age of 74.7 \pm 7.4 years were studied. Results - The main causes for intestinal obstruction were: adhesion (38\%), colon cancer (24\%), hernia (12\%), fecal impaction (12\%), carcinomatosis (10\%) and sigmoid volvulus (4\%). Mortality was of $18 \%$, being associated with infection complications in all patients. Conclusions - a) The distinction between functional and organic intestinal obstruction in elderly individuals is difficult; b) Colon cancer is an important cause of intestinal obstruction, since obstructive conditions may be the first clinical manifestation of such tumors; c) Adhesion and hernia are equally important in the etiology of bowel obstruction, the former so being in terms of incidence and the latter in relation to the possibility of developing bowel strangling; d) The clinical signs of obstruction are not evident in elderly individuals and leukocyte count can be more reliable for this diagnosis.

HEADINGS - Bowel/intestinal obstruction, elderly, treatment. 


\section{REFERÊNCIAS}

1. Abi-Hanna P e Gleckman R. Acute abdominal pain: a medical emergency in older patients. Geriatrics 1997; 52: 72-4.

2. Bevan PG. Adhesive obstruction. Ann R. Coll Surg Engl 1984; 66: 164-9.

3. Bizer LS, Liebling RW Delany HM, Gliedman ML. Small bowel obstruction. The role of nonoperative treatment in simple intestinal obstruction and predictive criteria for strangulation obstruction. Surgery 1981; 89: 407-13.

4. Brenner S, Campos GMR. Brenner AS, Schulz ES, Coelho JCU. Oclusão intestinal: análise de 276 casos. Rev Col Bras Cir 1994; 21 : 1-5.

5. Castell DO. Esophageal disorders in the elderly Gastroenterol Clin North Am 1990; 19: 235-54.

6. Chowbey PK Panse R, Sharma A, Khullar R, Soni V, Baijal M. Elective laparoscopy in diagnoses and treatment of recurrent small bowel obstruction. Surg Laparosc Endosc Percutan Tech 2006; 16: 416-22.

7. Dauphine CE, Tan P, Beart RW, Vukasin P, Cohen H, Corman ML. Placement of self-expanding metal stents for acute malignant large bowel obstruction: a collective review. Ann Surg Oncol 2002; 9: 574-9.

8. De Lillo AR e Rose S. Functional bowel disorders in the geriatric patient: constipation, fecal impaction and fecal incontinence. Am J Gastroint 2000; 95: 901-5.

9. Duron JJ, Jourdan-Da Silva N, Montcel ST, Berger A, Muscarí F, Hennet H, Veyrieres M, Hay JM. Adhesive postoperative small bowel obstruction: incidence and risk factors of recurrence after surgical treatment. A multi center prospective study. Ann Surg 2006; 244: 750-7.

10. Firth $\mathrm{M}$ e Prather CM. Gastrointestinal motility problems in the elderly patient. Gastroenterology 2002; 122: 688-700.

11. Galvão-Alves J e Couto RE. Doença do aparelho digestório do idoso - J Bras Gastroenterol 2007; 7: 68-74.

12. Gleckman R e Hilbert D. Afebrile bacteremia. A phenomenon in geriatric patients. JAMA 1982; 248: 1478-81.

13. Gold $\mathrm{S}$ e Bergman. A geriatric consultation team in the emergency department. J Am Geriatr Soc 1997;45:764-7.

14. Greene WW. Bowel obstruction in the aged patient. A review of 300 cases. Am J Surg 1969; 118:541-5.

15. Holt PR. Diarrhea and malabsorption in the elderly. Gastroenterol Clin North Am 2001; 30: 427-44.

16. Ihedioka U, Alani A, Modak P, Chong P, O' Dwyer PJ. Hernias are the most common cause of strangulation in patients presenting with small bowel obstruction. Hernia 2006; 10: 338-40.

17. Koruth NM, Hunter DC, Krukowski ZH, Matheson NA. Immediate resection in emergency large bowel surgery: a 7 year audit Br J Surg 1985; 72: 703-7.

18. Koruth NM, Krukowski ZH, Yoringson GG, Hendry WS, Logic JRC, Jones $\mathrm{PF}$, Munro A. Intra-operative colonic irrigation in the management of left-sided large bowel emergencies. Br Surg 1985; 72: 1472-79.
19. Lebrão ML. O idoso no Brasil: aspectos da transição demográfica e epidemiológica. In: Cerqueira ATAR e Oliveira NIL. Compreendendo e cuidando do idoso: uma abordagem multi-profissional, 2006. Conselho Editorial da Faculdade de Medicina de Botucatu .p 19-31.

20. Lee YM, Law WL, Chu KW, Poon RTP. Emergency surgery for obstructing colorectal cancers: a comparison between right-sided and left-sided lesion. J Am Coll Surg 2001; 192: 719-25.

21. Markogiannakis H, Messaris E, Dardamanis D, Pararas N, Tzertzemelis D, Grannopoulos P Larentzabis A, Lagoudianakis E, Manouras A, Bramis I. Acute mechanical bowel obstruction: clinical presentation etiology, management and outcome. World J Gastroenterol 2007; 13: 432-7.

22. Miller G. Boman J, Shrier I, Gordon PH. Etiology of small bowel obstruction. Am J Surg 2000; 180: 33-6.

23. Miyauchi T, Kuroda T, Nisioka M, Hashimoto T, Kasamutu T, Kuratate S, Fujimine M. Clinical study of strangulation obstruction of the small bowel J Med Investig 2001; 48: 66-42.

24. Moreira Jr. H, Moreira H, Isaac RR, Fernandes ACAC, Mascarenhas JCS, Moreira JPT. Obstrução intestinal: análise retrospectiva da etiologia e morbimortalidade no hospital de urgências em Goiânia (HUGO). Rev Bras Coloproct 2005, 25: 137-45.

25. Nagle A, Ujiki M, Denham W, Murayama K. Laparoscopic adhesiolysis for small bowel obstruction. Am J Surg 2004; 187: 464-70.

26. O’Boyle CJ, Mac Fie J, Mitchell CJ, Mac Fie J, Mitchell CJ, Johnstone D, Sagar PM, Selmans PC. Microbiology of bacterial translocation in humans. Gut 1998; 42: 29-35.

27. Organización mundial de la Salud. Grupo científico sobre la epidemiologia del envejecimiento, Ginebra: OMS; 1984[ serie de informes técnicos, 706].

28. Petroianu A, Sabino LO, Oliveira Neto JE, Marques MS. Principais causas de obstrução intestinal mecânica de tratamento cirúrgico, em crianças e em adultos. Folha Medica 1994; 109: 53-5.

29. Rodrigues - Paz CA e Palacio-Velez F. Causas y, mortalidade de la obstrucción intestinal en el anciano. Rev. Gastroenterol Mex 2000; 65: 121-3.

30. Rosenthal RA e Zenilman ME. Cirurgia no idoso. In: Sabiston T. Tratado de cirurgia. As bases biológicas da prática cirúrgica moderna. Tow send CM ed Guanabara Koogan 2002, p. 243-66.

31. Soergel K Zboraiske F, Amberg. Presbyesophagus: Esophageal motility in nonagenarians. J Clin Invest 1964; 43: 1472-79.

32. Talley NG, Fleming KC, Evans JM O'Keefe EA, Weaver AL, Zinsmeister AR, Melton L. Constipation in an elderly community: a study of prevalence and potencial risk factors. Am J Gastroenterology 1996; 91: 19-25.

33. Zadeh BJ, Davis JM, Canizaro PC. Small bowel obstruction in the elderly. Am Surg 1985;51: 470-3.

Conflito de interesse: não há

Fonte financiadora: não há

Recebido para publicação em: 15/07/2007 Aceito para publicação em: 20/10/2007 